Article

\title{
A Flexible Strain Sensor Based on the Porous Structure of a Carbon Black/Carbon Nanotube Conducting Network for Human Motion Detection
}

\author{
Peng Zhang, Yucheng Chen, Yuxia Li, Yao Zhang, Jian Zhang and Liangsong Huang * \\ Key Laboratory for Robot Intelligent Technology of Shandong Province, Shandong University of Science and \\ Technology, Qingdao 266590, China; pengzhang@sdust.edu.cn (P.Z.); chenyucheng@sdust.edu.cn (Y.C.); \\ yuxiali2004@sdust.edu.cn (Y.L.); zhangyao@sdust.edu.cn (Y.Z.); zhangjian@sdust.edu.cn (J.Z.) \\ * Correspondence: lshuang@sdust.edu.cn
}

Received: 3 December 2019; Accepted: 18 February 2020; Published: 20 February 2020

check for updates

\begin{abstract}
High-performance flexible strain sensors are playing an increasingly important role in wearable electronics, such as human motion detection and health monitoring, with broad application prospects. This study developed a flexible resistance strain sensor with a porous structure composed of carbon black and multi-walled carbon nanotubes. A simple and low-cost spraying method for the surface of a porous polydimethylsiloxane substrate was used to form a layer of synergized conductive networks built by carbon black and multi-walled carbon nanotubes. By combining the advantages of the synergetic effects of mixed carbon black and carbon nanotubes and their porous polydimethylsiloxane structure, the performance of the sensor was improved. The results show that the sensor has a high sensitivity (GF) (up to 61.82), a wide strain range (0\%-130\%), a good linearity, and a high stability. Based on the excellent performance of the sensor, the flexible strain designed sensor was installed successfully on different joints of the human body, allowing for the monitoring of human movement and human respiratory changes. These results indicate that the sensor has promising potential for applications in human motion monitoring and physiological activity monitoring.
\end{abstract}

Keywords: flexible strain sensor; carbon black; multi-walled carbon nanotubes; human motion detection

\section{Introduction}

In recent years, various flexible strain sensors which convert mechanically-dependent variables (such as stretching [1], bending [2], and torsion [3]) into electrical signals have been developed. Applications of such sensors include their use as wearable, soft sensor joints on the surface of the skin, which can measure the biological and physiological activities of the user. These advantages and developments have attracted growing attention and applications in artificial intelligence [4,5], human motion detection [6-8], human facial expression recognition [9,10], intelligent robots [11,12], and health monitoring [13,14], among others [15]. However, in view of the widespread application of these sensors, several important requirements and considerations need to be met. In order to better monitor the complex signal, the strain sensor should have an adequate flexibility, high sensitivity, large measuring range, fast response ability, excellent durability, and small volume. As well as being comfortable and convenient to wear, easy integration with an external circuit and a low production cost are also key factors that need to be considered. At present, to satisfy the aforementioned characteristics, researchers have designed strain sensors with different sensing mechanisms, among which the resistance sensor has gradually become the mainstream design method of strain sensors due to its simplicity and low cost. According to the response principle of resistance sensors, conductive carbon 
fillers and mental nanowires (such as carbon black [16,17], carbon nanotubes [18,19], graphene [20,21], and Ag nanowires [22,23]) can be combined with flexible substrates (such as polydimethylsiloxane (PDMS) [24,25], polyurethane (PU) [26,27], silicone rubber (SR) [28,29], elastic fabrics [30,31], and elastic bands [32]) to make a flexible piezoresistive sensor with a high sensitivity and a large stretch range by a certain preparation method. Therefore, these studies reveal that composites made of a conductive material and polymer can meet the performance requirement of strain sensors. In addition, the reasonable fabrication costs and the excellent performances obtained from sensors based on conductive carbon filling materials have made carbon nanotubes and carbon black the best conductive materials for many scholars $[33,34]$.

Carbon black $(\mathrm{CB})$ is a kind of zero-vinami material with a low aspect ratio and large surface area. The conductive channel formed by the contact between carbon black particles can exhibit great changes under low strain levels. However, under higher strain, the gap between carbon black particles increases, which prevents the formation of an effective conductive channel and thus leads to a reduced sensitivity at high levels of strain [35]. As a one-dimensional cylindrical nanostructure, carbon nanotubes (MWNTS) have significant electrical and mechanical properties, and the contact between them can form a close conductive network [36,37]. Due to the light connection between carbon nanotubes, the conductive network formed by carbon nanotubes will not change significantly under low strain, and its resistance only marginally changes. The resistance does not change appreciably until a large strain is applied that destroys the conductive network. Therefore, by combining the characteristics of carbon black and carbon nanotubes, a collaborative conductive network can be established by mixing [34] or layering [38] the two conductive carbon fillers, which not only reduces the production cost, but also significantly improves the electrical characteristics of nano-composite materials. Zheng et al. prepared a strain sensor composed of CB-MWNTs-PDMS mixed in solution, which had a stretching range of up to $300 \%$. Although the sensor could detect human joint movement reasonably well, the monitoring of micro-strain proved to be difficult. Additionally, the large volume of the sensor caused notable discomfort to the user [33]. A layer-by-layer sensor assembly was proposed by Zhang et al., which involved the addition of carbon black and carbon nanotubes to a yard. The sensor was characterized by a simple operation, good linearity, and sensitivity $(G F=45.4)$, but the conductive material was blocked by Polyvinyl alcohol (PVA) and the synergistic effects of carbon black and carbon nanotubes were not well-exhibited [38].

In this study, in order to improve the sensitivity, reduce the size of the sensor, and increase the wearability, we developed a strain sensor with a high sensitivity, good linear response, large stretch range, and good reliability. Due to its water-soluble properties, granulated sugar was mixed with PDMS to form a stretching matrix with a porous structure after immersion. The experimental data show that the porous structure can significantly improve the stretching range of the sensor to up to 1.3 times its own length. Combined with the unique properties of conductive carbon fillers, such as carbon black and multi-walled carbon nanotubes (MWNTs), a conductive network layer is can be formed on the porous substrate surface through a simple and low-cost spraying method, which improves the sensitivity of the sensor. The flexible strain sensor prepared by this method can monitor the movement of facial muscles and joints, including fingers, wrists, elbows, and knees, as well as other deformations of different magnitudes. In addition, the monitoring of human physiological activities, such as breathing and other minor deformations, can be achieved. These results show that the sensor is a simple, cost-effective solution suitable for large-scale production, with a broad application prospect for wearable monitoring devices. A comparison of the sensor developed in this paper and the characteristics of those proposed in [33] and [38] is shown in Table 1. The variations show evident differences in sensor performance arising from varying methods of preparation.

The specific fabrication method and the process of the flexible strain sensor are discussed in Section 2. Furthermore, Section 3 presents the various sensing characteristics of the flexible strain sensor and analyzes the test results. Finally, a summary and conclusion for the present study are presented in Section 4. 
Table 1. Comparisons of the performances of flexible strain sensing devices.

\begin{tabular}{|c|c|c|c|c|c|}
\hline Reference & $\begin{array}{l}\text { Creation } \\
\text { Method }\end{array}$ & $\begin{array}{l}\text { Conductive } \\
\text { Material }\end{array}$ & Gauge Factor & Linear Range & Characteristics \\
\hline [33] & $\begin{array}{l}\text { Solution } \\
\text { mixture }\end{array}$ & $\begin{array}{c}\text { CB } \\
\text { MWNTS }\end{array}$ & $\begin{array}{c}0 \%-100 \%-0.91 \\
100 \%-255 \%-3.25 \\
255 \%-300 \%-13.1\end{array}$ & $0 \%-300 \%$ & $\begin{array}{l}\text { Insensitivity to small strain, } \\
\text { inadequate comfort }\end{array}$ \\
\hline [38] & $\begin{array}{l}\text { Layer-by-layer } \\
\text { assembly }\end{array}$ & $\begin{array}{c}\text { CB } \\
\text { MWNTS }\end{array}$ & Maximum to 45.4 & $15 \%-150 \%$ & $\begin{array}{c}\text { Conduction synergy } \\
\text { inefficiency }\end{array}$ \\
\hline This work & Spraying & $\begin{array}{c}\text { CB } \\
\text { MWNTS }\end{array}$ & $\begin{array}{c}0 \%-40 \%-16.12 \\
40 \%-130 \%-61.82\end{array}$ & $0 \%-130 \%$ & $\begin{array}{c}\text { Sensitivity to small strain, } \\
\text { high sensitivity }\end{array}$ \\
\hline
\end{tabular}

\section{Design and Fabrication}

\subsection{Experimental Materials}

In this experiment, high-purity multi-wall carbon nanotubes (MWNTs) were provided by Nanjing XANANO Materials Tech Co., Ltd., Nanjing, Jiangsu Province, China. The purity of the MWNTs was $95 \%$, with a diameter of $10-20 \mathrm{~nm}$, a length of $10-30 \mu \mathrm{m}$, and a carboxyl content of $2.00 \mathrm{wt} \%$. Carbon black (CB) of the model BP2000 was purchased from Cabot Corporation, Boston, Massachusetts, USA. Alcohol was used as the solvent of conductive carbon fillers, which can accelerate the evaporation of the solvent. The mixture of $\mathrm{CB} / \mathrm{MWNTs}$ was added to the alcohol for three hours of ultrasonic mixing, and a CB/MWNT dispersion with a mass fraction of $2.00 \mathrm{wt} \%$ (1.00 $\mathrm{wt} \%$ each for CB and MWNTs) was obtained [33]. PDMS was used as the matrix material (Sylgard 184; Dow Corning Corp., Gales Ferry, Connecticut, USA), and the prefabricated mixture was prepared using a 1:15 mass ratio of the curing agent to the substrate, which was stirred for $10 \mathrm{~min}$ and set aside. Granulated sugar was ground in the grinder for $30 \mathrm{~min}$ for later addition to the matrix.

\subsection{Fabrication Process of the Porous Flexible Strain Sensor}

The fabrication process of the porous flexible strain sensor is shown in Figure 1. First, as shown in Figure 1a, the substrate of the porous flexible strain sensor was prepared by the blending method. Mixtures with mass fraction ratios of $m$ (sugar) $/ m$ (PDMS) $=n$ (where $n=0 \%, 10 \%, 20 \%, 30 \%, 40 \%$ or $50 \%$ ) were stirred magnetically for $2 \mathrm{~h}$ to obtain evenly dispersed sugar/PDMS mixtures. Then, the excess bubbles were removed by vacuum treatment for $20 \mathrm{~min}$, and the various mixtures with different mass fraction ratios were poured into cylindrical molds with an inner diameter of $2.25 \mathrm{~mm}$ and cured in an oven at $80^{\circ} \mathrm{C}$ for $3 \mathrm{~h}$. The substrate of the cured sugar/PDMS mixture was then taken out and placed in distilled water for $48 \mathrm{~h}$ of ultrasound treatment to dissolve the granulated sugar within the cured sugar/PDMS mixture completely, and to obtain the porous PDMS substrate, as indicated in Figure 1b. Mechanical tests were conducted on the porous substrate with different mass fraction ratios and the substrate with the best tensile properties was selected (see Section 3.1 for details). The selected substrate was then put into an ethanol solution for $10 \mathrm{~min}$ of ultrasonic cleaning to remove any stains and dust on the surface in Figure 1c. After drying, the substrate was rinsed in an oxygen plasma cleaner for $90 \mathrm{~s}$, as shown in Figure 1d. The purpose of this was to improve the surface activity of PDMS by making it hydrophilic and to increase the absorption of conductive nano-materials. Finally, the conductive composite mixture was sprayed evenly on the porous substrate using a spray bottle, and the porous flexible strain sensor was obtained after drying in the oven, as presented in Figure 1e.

\subsection{Characterization and Electrical Measurements}

The porous flexible strain sensor was characterized by scanning electron microscopy (SEM), and the results are shown in Figure 2. The micrograph of Figure 2a shows evident irregular pores inside the PDMS substrate, indicating that the porous effect was achieved and that a conductive network layer was wrapped around the PDMS substrate. By further magnification of the conductive material coating on the sensor surface, it was revealed that the close contacts of CB and MWNTS crisscross together to form a cooperative conductive network, as exhibited in Figure $2 \mathrm{~b}$. The conductive channel 
was mainly formed by CB-CB, CB-MWNT, and MWNT-MWNT connections (indicated by the yellow circle in Figure $2 b$ ), which suggests that the prepared material achieved the desired effect.

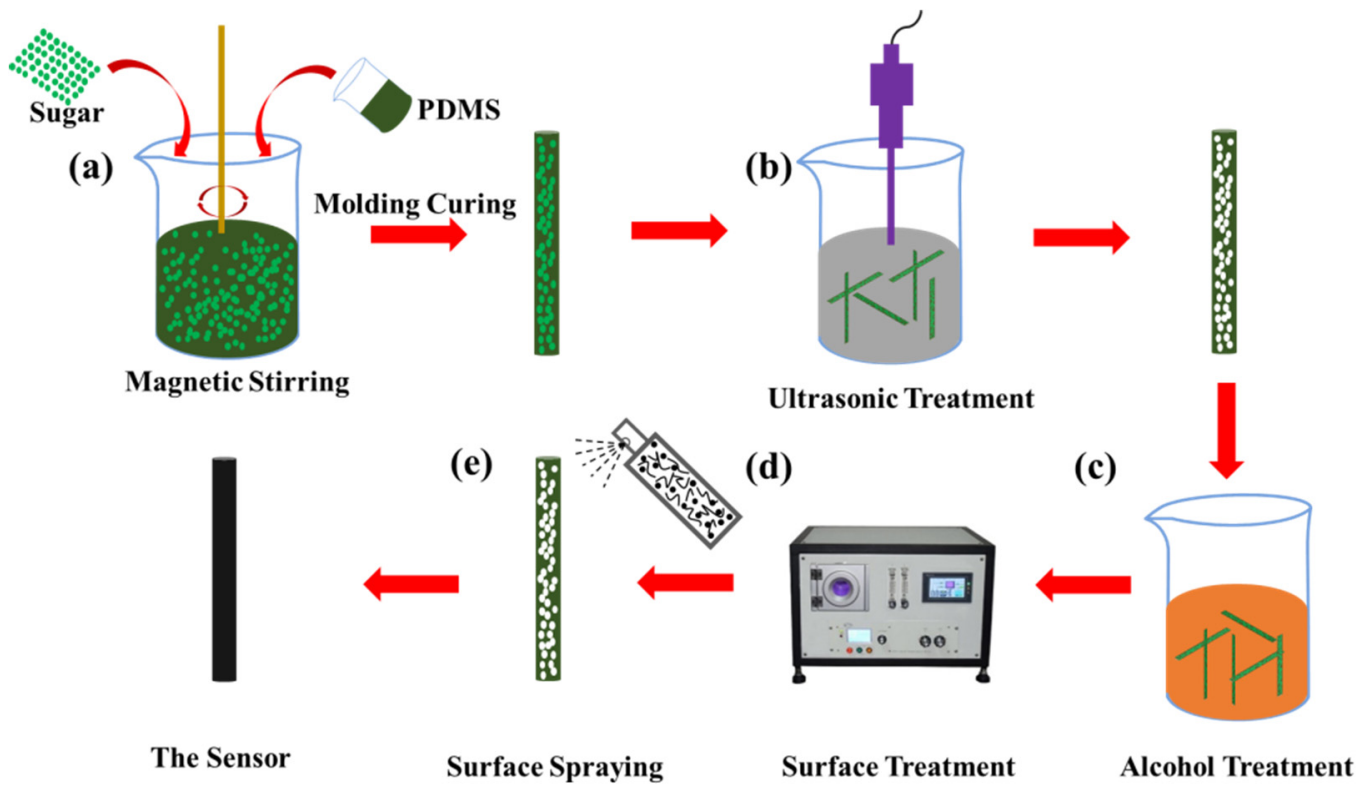

Figure 1. The preparation process of the flexible strain sensor: (a) matrix preparation by solution mixing; (b) ultrasonic treatment of the matrix in distilled water employed to obtain the porous structure; (c) cleaning of the matrix using alcohol; (d) the surface of the matrix is treated with isoxic ions to improve the surface activity; (e) strain sensors obtained by spraying.
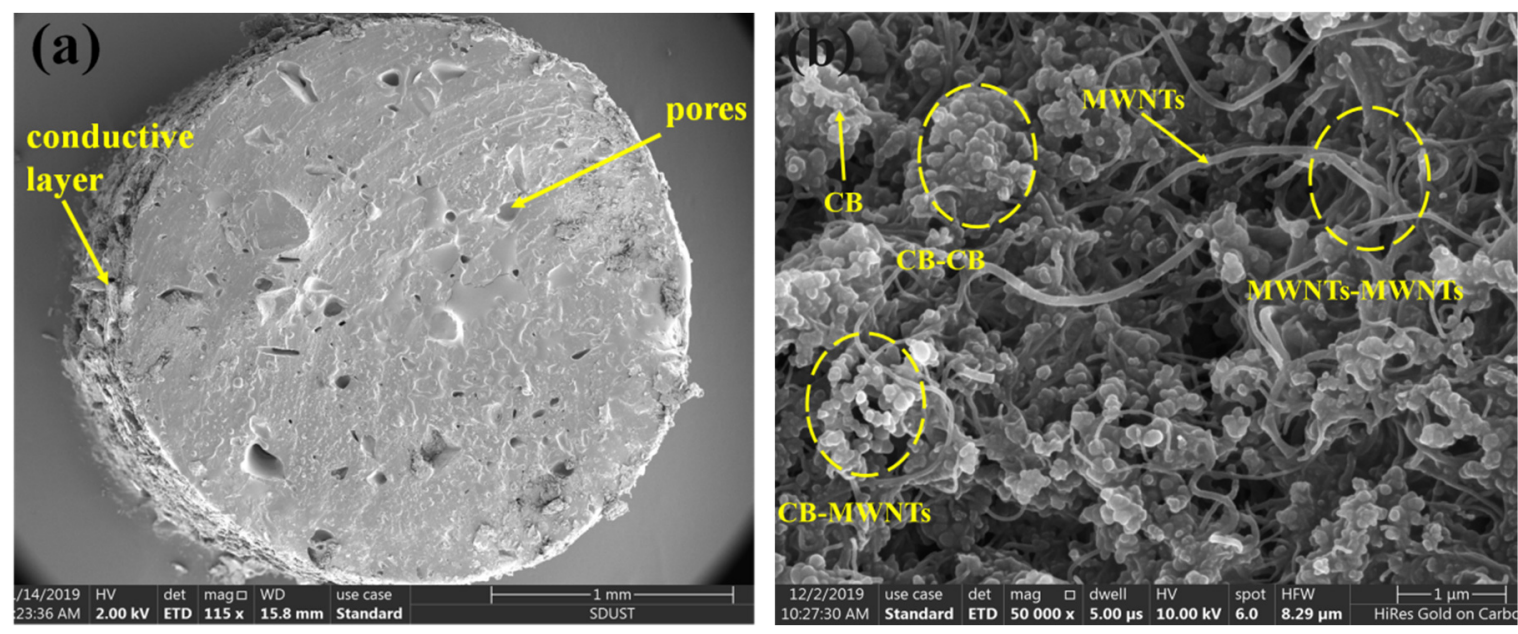

Figure 2. Scanning electron microscopy (SEM) diagram of the flexible strain sensor: (a) SEM diagram of the sensor, the porous structure inside the matrix, and the conductive layer on the surface of the matrix and (b) SEM image of the conductive network on the sensor surface and the three conductive connection channels (carbon black (CB)-CB, CB-carbon nanotubes (MWNTS), and MWNTS-MWNTS).

The experimental setup of the sensor testing process is detailed in Figure $3 a, b$. By setting the tension gauge to apply strain in different directions, forces were applied to the sensor in both stretching and compressing directions to test the performance parameters of the sensor. The tension gauge was the ZQ-990A model from China Smart Instrument Co., Ltd., with a measuring range of $0 \sim 50 \mathrm{~N}$ and a force resolution ratio of $0.01 \mathrm{~N}$ (Figure 3). The resistance parameter testing instrument was a desktop LCR meter (TH2826) from Changzhou Tonghui Electronics Co., Ltd. As shown in Figure 3a, during the testing of tensile properties, both ends of the strain sensor were fixed firmly to the fixture. During the 
testing of bending characteristics, the strain sensor was fixed on the PET substrate, which is shown in Figure 3b. A temperature measuring instrument (303-0a, Shanghai kuntian laboratory instrument Co., Ltd.) was used to record the temperature of the sensor.

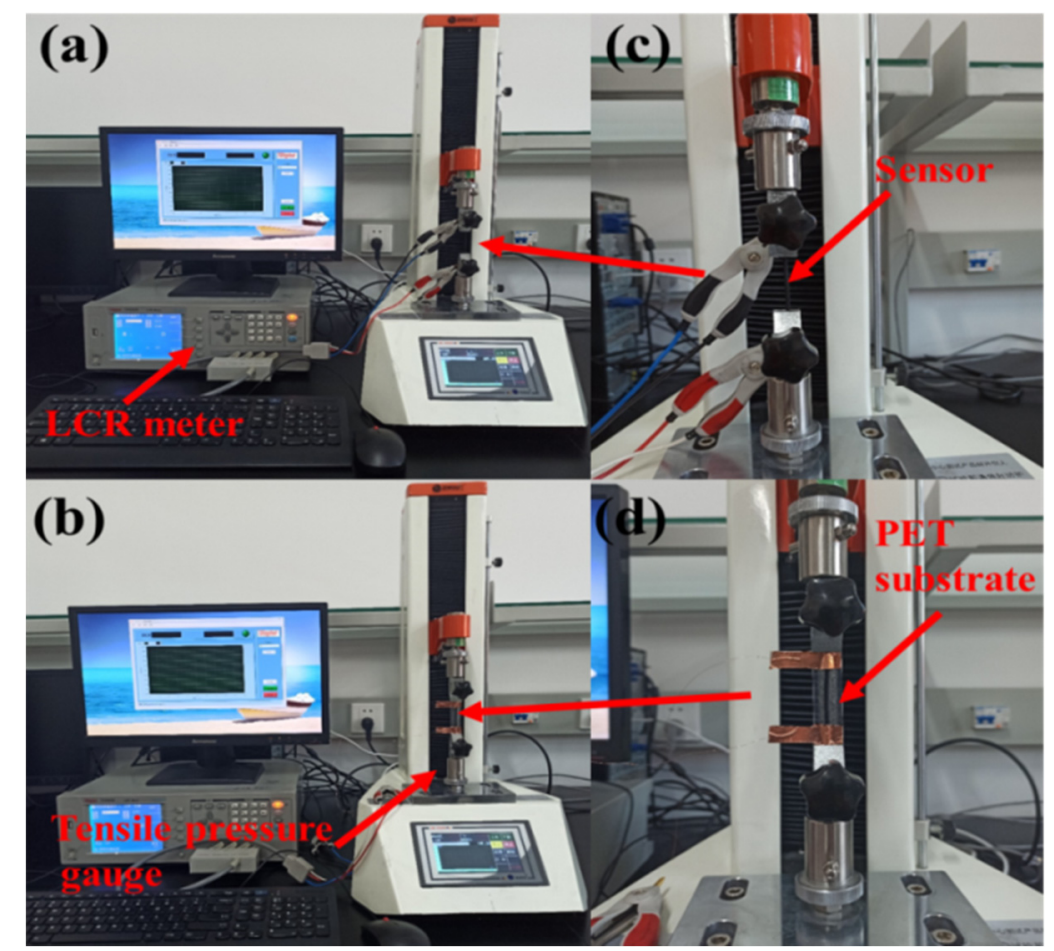

Figure 3. Experimental setup for sensor testing: (a) tensile properties testing process diagram; (b) bending properties testing process diagram; (c) a partially enlarged view of (a); (d) a partially enlarged view of (b).

\section{Results and Discussion}

\subsection{Characteristics of the Flexible Strain Sensor}

The mechanical properties of the six groups of flexible strain sensor samples with mass fraction ratios of $m$ (Sugar) $/ m($ PDMS $)=n(n=0 \%, 10 \%, 20 \%, 30 \%, 40 \%$ or $50 \%)$ were evaluated by tensile and compression tests. Figure 4 shows the stress-strain curve and mechanical properties of the six groups of samples. The results of Figure $4 \mathrm{a}$ indicate that the porous structure created by sugar dissolution can change the elastic modulus of the material and increase the tensile length of the matrix, thus improving the tensile performance of the sensor. When the mass fraction ratio is $30 \mathrm{wt} \%$, the sensor exhibits the highest variation in tensile strain of up to approximately $130 \%$. Detailed mechanical properties are shown in Figure $4 \mathrm{~b}$, where it is evident that at a mass fraction ratio of $30 \mathrm{wt} \%$, the corresponding PDMS substrate has the highest tensile strength and elongation at the breaking point. Therefore, in the subsequent experiments, the PDMS substrate with a mass fraction ratio of $30 \mathrm{wt} \%$ was selected as the experimental sample.

The sensitivity (GF) formula of the strain sensor is as follows:

$$
G F=\left(\Delta R / R_{0}\right) / \varepsilon
$$

where $\Delta R$ is the resistance variation under strain $\left(\Delta R=R-R_{0}\right), R_{0}$ is the initial resistance without strain, and $\varepsilon$ is the relative variation in the length of the sensor under strain. 

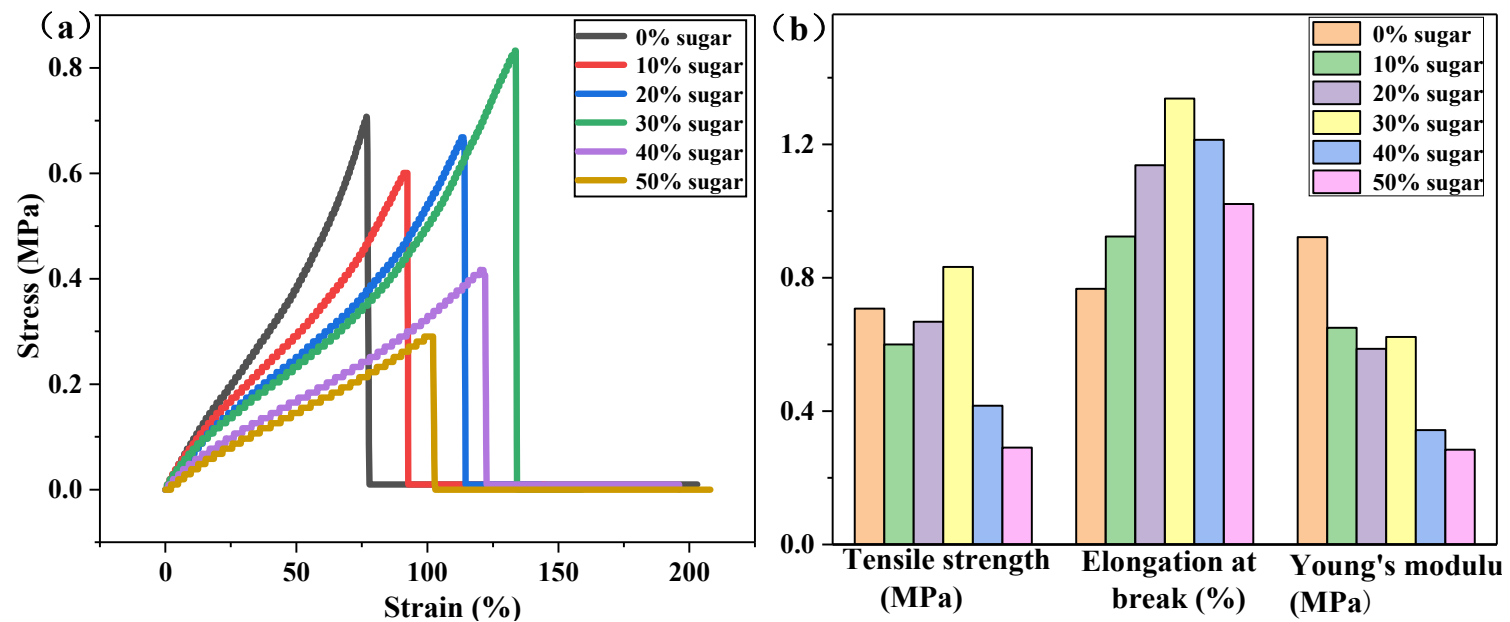

Figure 4. The stress-strain curve and mechanical properties of the sensors with different mass fraction ratios: (a) the stress-strain curve of the sensor; (b) the mechanical performance diagram of the sensor.

The changes in sensor resistance during the strain process are shown in Figure 5. When the sensor is not stretched, no cracks appear on the substrate surface, and there is a close connection between CB-CB, CB-MWNTS, and MWNTS-MWNTS, which forms a large number of conductive pathways (the red curves show conductive pathways in Figure 5). Subsequently, when the sensor is subjected to a small tension force, the substrate exhibits inconspicuous surface cracks, which can be described as larger gaps between carbon black particles in the microscopic structure. Although the separation between CB particles (the yellow circles in Figure 5) reduces the conductive pathways, the connections between CB-MWNTS and MWNTS-MWNTS are affected minimally, and the variation in resistance is thus small. Furthermore, at larger tension forces, obvious cracks appear on the surface of the substrate and the gaps have been widened, resulting in a large number of fractures within the conductive pathways between CB and MWNTS, and between MWNTS (the blue circles in Figure 5). As such, the change in resistance of the sensor increases significantly.
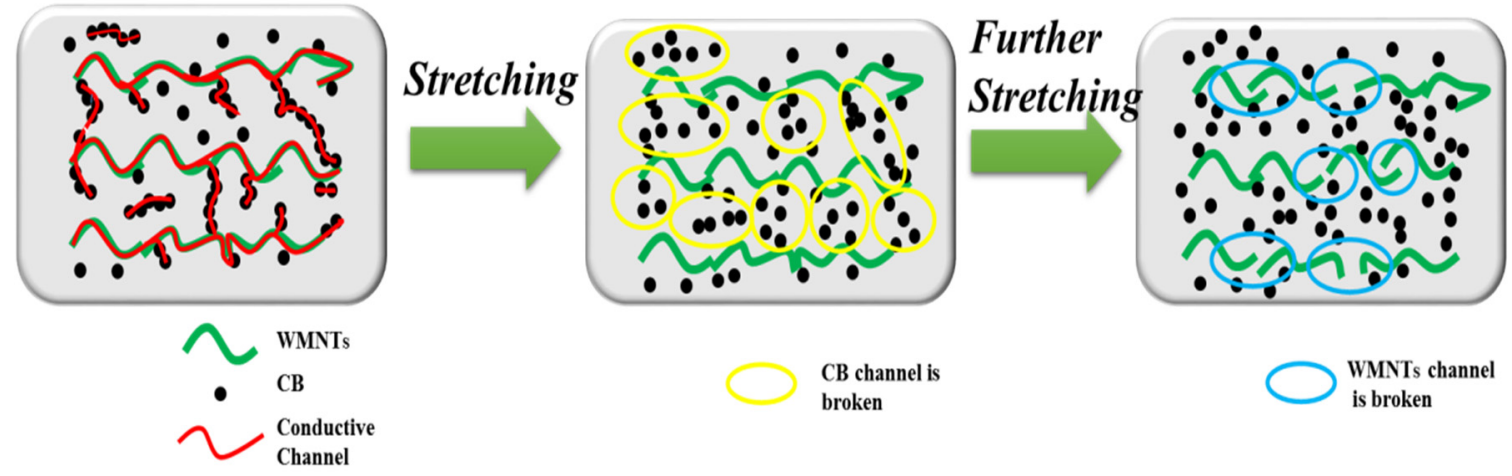

Figure 5. Schematic diagram of the conductive network path of the flexible strain sensor and evolution of the conductive network path under stretching.

The relationship between the variation in resistance $\Delta R / R_{0}$ and tensile elongation $(0 \%-130 \%)$ of the sample is shown in Figure 6a. The variation in resistance $\Delta R / R_{0}$ of the strain sensor demonstrates evident monotonous increases as the tensile length is varied. According to the trends of the curve, the GF of the sensor can be divided into two stages: at tensile strains ranging from $0 \%$ to $40 \%$, the GF is 16.12 , while higher levels of tensile strain between $40 \%$ and $130 \%$ are characterized by a GF of 61.82 . These values show that the strain sensor can be adapted to the different tension ranges. In order to verify the dynamic characteristics of the strain sensor, the sensor was stretched by $2 \%, 25 \%, 50 \%$ and $75 \%$ of its own length, and 10 stretch cycles were performed. The results shown in Figure $6 \mathrm{~b}$ reveal that 
the $\Delta R / R_{0}$ changes with the periodic changes in tension force and the sensor has a good regularity and stability. The sensor was held under tensile strain for $70 \mathrm{~s}$ in order to verify the stability of the sensor under a prolonged state of tension. Furthermore, Figure $6 \mathrm{c}$ shows that the $\Delta R / R_{0}$ tends to be stable after an overshoot peak (overshoot recovery time: $2 \mathrm{~s}, 5 \mathrm{~s}, 10 \mathrm{~s}, 14 \mathrm{~s}$ and $17 \mathrm{~s}$ ), suggesting that the sensor recovers quickly and is able to perform reliably following the overshoot. This overshoot may be due to the acceleration caused by the tension meter as the sensor is stretched. At the same time, the influence of the tensile rate on the strain sensor was also investigated and the results are presented in Figure $6 \mathrm{~d}$. The test results show that at a tensile strain of $30 \%$, as the tensile rate increases incrementally from $10 \mathrm{~mm} / \mathrm{min}$ to $100 \mathrm{~mm} / \mathrm{min}$, the tensile rate has no obvious effect on the $\Delta R / R_{0}$ of the strain sensor. This indicates that under external stresses at different frequencies, the sensor remains stable and can meet the needs of motion detection. The tests were repeated 100 times by loading and unloading the tension variation on the sensor, which was $2 \%$ and $50 \%$, to study the robustness and reliability of the sensor under continuous strain. The $\Delta R / R_{0}$ of the sensor under repeated loading at two different levels of tension is shown in Figure 6e,f. The changes observed for the resistance wave form are similar to those of cyclic loading. At the end of the test, the waveform did not show obvious attenuation, which suggests that the sensor maintained an excellent reliability under the action of a repeated strain force. Figure $6 \mathrm{~g}$ shows that the sensor resistance increases with increasing temperature and decreases as the temperature is reduced. This is due to the thermal expansion of the conductive composite layer with the increase of temperature, and the distance between the conductive fillers increases, resulting in the increase of resistance. In contrast, when the temperature decreases, the conductive composite layer shrinks and the distance between the conductive fillers decreases, resulting in a reduction in resistance. As the temperature increases from 22 to $51{ }^{\circ} \mathrm{C}$, the $\Delta R / R_{0}$ does not exceed $10 \%$, which basically meets the needs of high-temperature conditions.

Finally, the bending performance of the strain sensor was investigated by attaching the sensor to a PET substrate, which was when installed on the fixture of the bending test instrument in Figure 7. The cyclic compression test was conducted by setting the procedure loop shown in Figure 6h, with the angle of bending set to $30^{\circ}$ for $45^{\circ}$ repetitions. From the test results below, it can be seen that the resistance of the strain sensor increases during bending, and is restored to its original value after it is released. This illustrates the resilience of the sensor under repeated bending and can meet the needs of bending measurements.
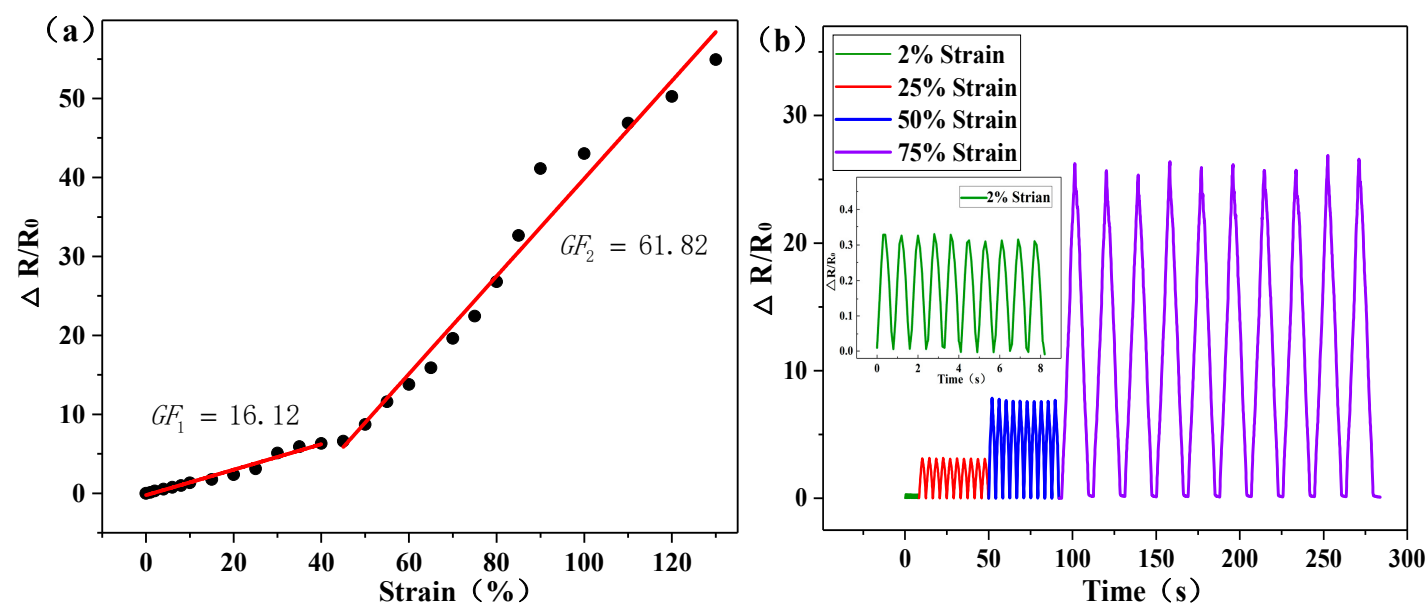

Figure 6. Cont. 

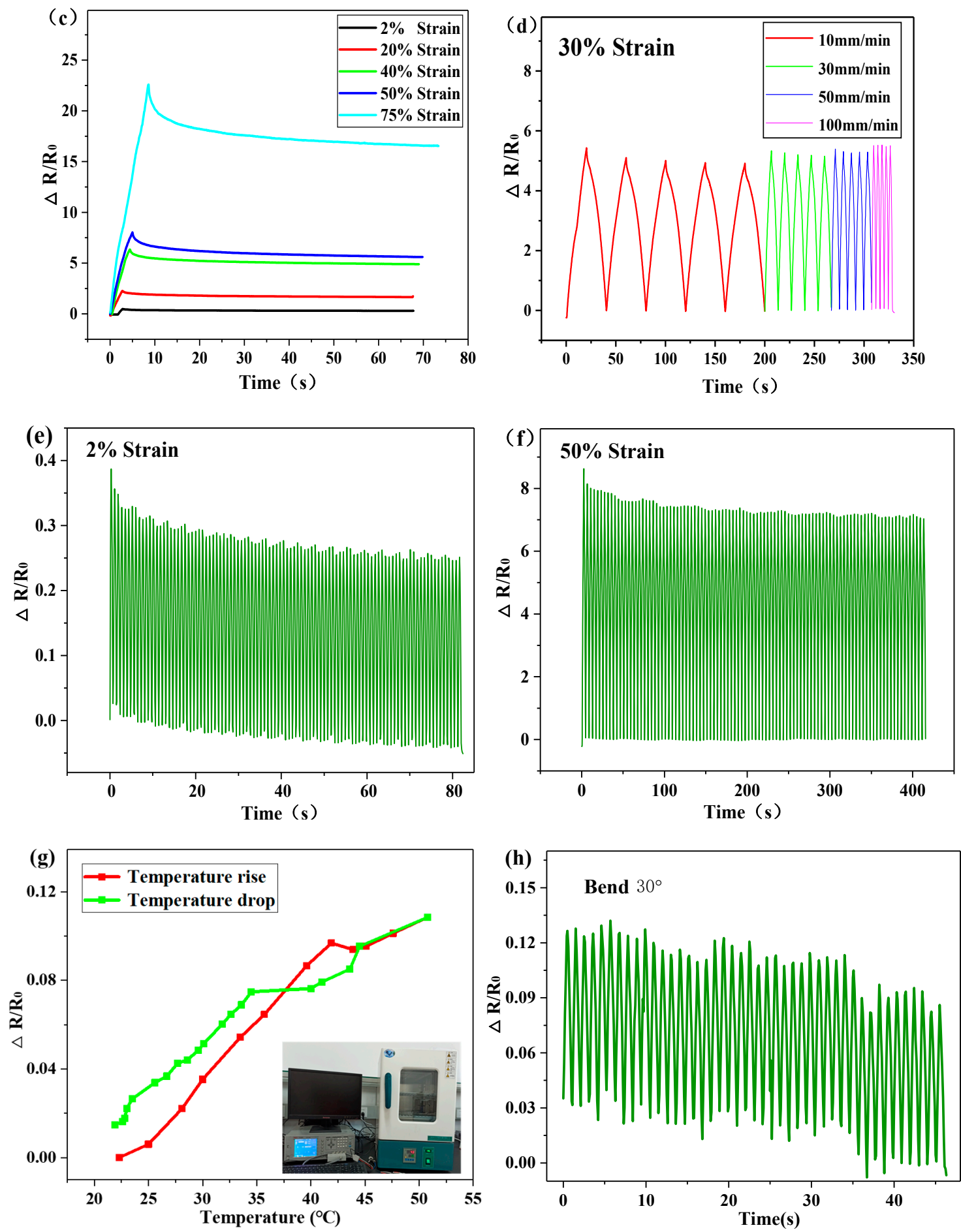

Figure 6. Performance tests of the flexible strain sensor: (a) changes in resistance with variations in strain; (b) multi-cycle testing of the sensor at different strain levels ( $2 \%, 25 \%, 50 \%$ and $75 \%)$; (c) sensing performance of the sensor at different strain rates at a strain of 30\%; (d) relative resistance variation for step strain; (e) relative resistance variation curve of the sensor for 100 consecutive cycles at a strain of $2 \%$; (f) relative resistance variation curve of the sensor for 100 consecutive cycles at a strain of $50 \%$; (g) relative resistance variation of the sensor at varying temperatures; (h) the relative change in resistance in a cyclic bending test. 


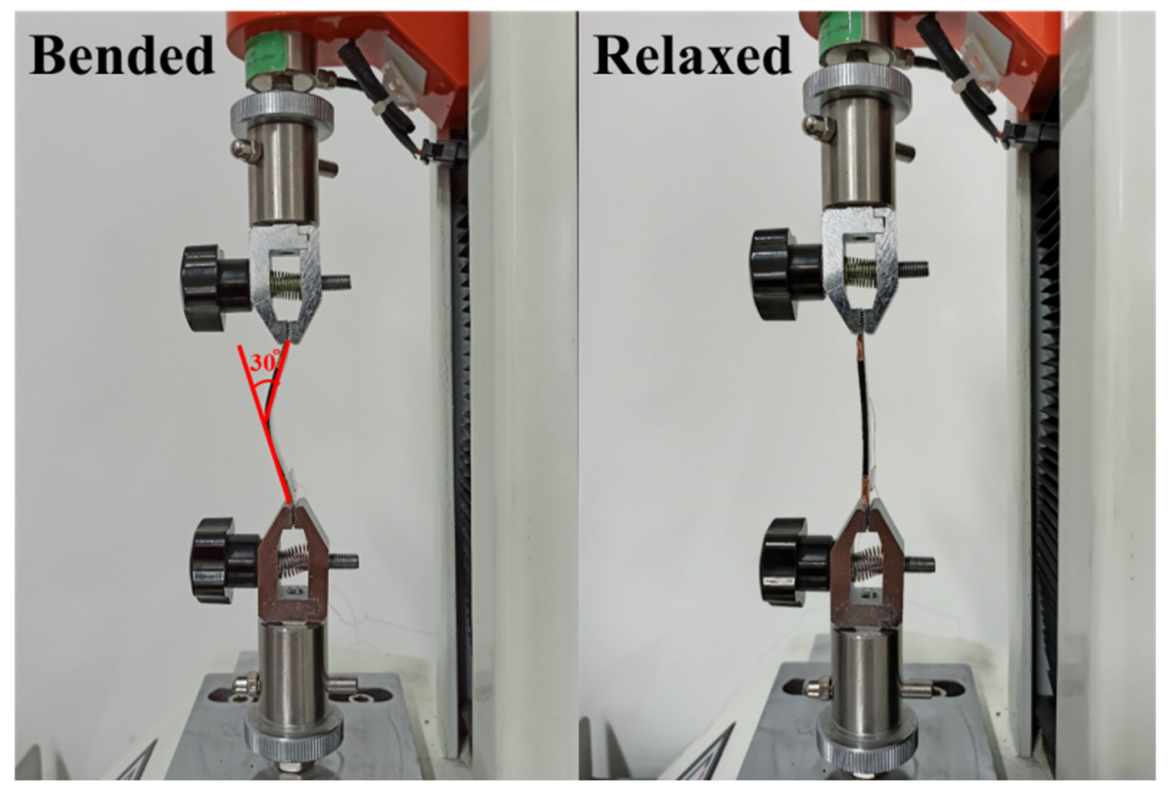

Figure 7. Bending test diagram of the flexible strain sensor on a PET substrate.

\subsection{Application of Strain Sensors as Wearable Strain Sensors for Human Motion Detection}

At present, wearable strain sensors have broad potential for applications in human body information detection due to their ability to respond to external mechanical stimulation. To verify that the sensor presented in this study can indeed be used as a wearable strain sensor, the sensor was attached to different parts of the body for testing. First, the sensors were attached to different joints of the body, and monitored the joint movements of the body in real time, according to the resistance variation. Figure 8a shows a flexible strain sensor on the index finger joint. As the finger joint periodically bends and extends at angles of $45^{\circ}$ and $90^{\circ}$, similar periodic changes in $\Delta R / R_{0}$ can be detected. When the finger joint is bent, the strain sensor is in the tensile state, and the $\Delta R / R_{0}$ increases. Similarly, when the finger joint returns to its initial state, the sensor reverts to its original position, and the $\Delta R / R_{0}$ subsequently decreases. Generally, the greater the degree of bending, the greater the deformation of the sensor and the greater the $\Delta R / R_{0}$. As is shown in Figure $8 \mathrm{~b}, \mathrm{c}$, the strain sensor was also attached to the wrist and elbow joints of the body. It can be observed that when the wrist joint (Figure $8 \mathrm{~b}$ ) and elbow joints bend at angles of $45^{\circ}$ and $90^{\circ}$ (Figure $8 \mathrm{c}$ ), the sensor reacts quickly, demonstrating that the strain sensor is sensitive to apparent strain and has a stable repeatability. Furthermore, the sensor was then attached to the knee to provide feedback on human movement monitoring. As shown in Figure $8 \mathrm{~d}$, the greater the bending degree, the greater the deformation of the sensor and the greater the $\Delta R / R_{0}$ increases with the bending angle of the knee when the knee is repeatedly flexed at $45^{\circ}$ and $90^{\circ}$. Additionally, Figure 8e shows repeated changes in $\Delta R / R_{0}$ during multiple half squats and full squats. The results of knee joint movement detection recorded while volunteers were walking and running are presented in Figure 8f,g. Figure 8g's results show that the $\Delta R / R_{0}$ changes with the cycle of walking steps, and the frequency of the waveform changes in line with human walking habits. Figure 8 h shows the change trend of the $\Delta R / R_{0}$ during the jogging and fast running of volunteers. We can see that the frequency of waveform change during fast running is significantly higher than that during jogging, which is consistent with the actual running situation. This indicates that the sensor can detect the running state. The experimental findings above suggest that the sensor can detect the state of running and can be used to monitor human joint movement. 

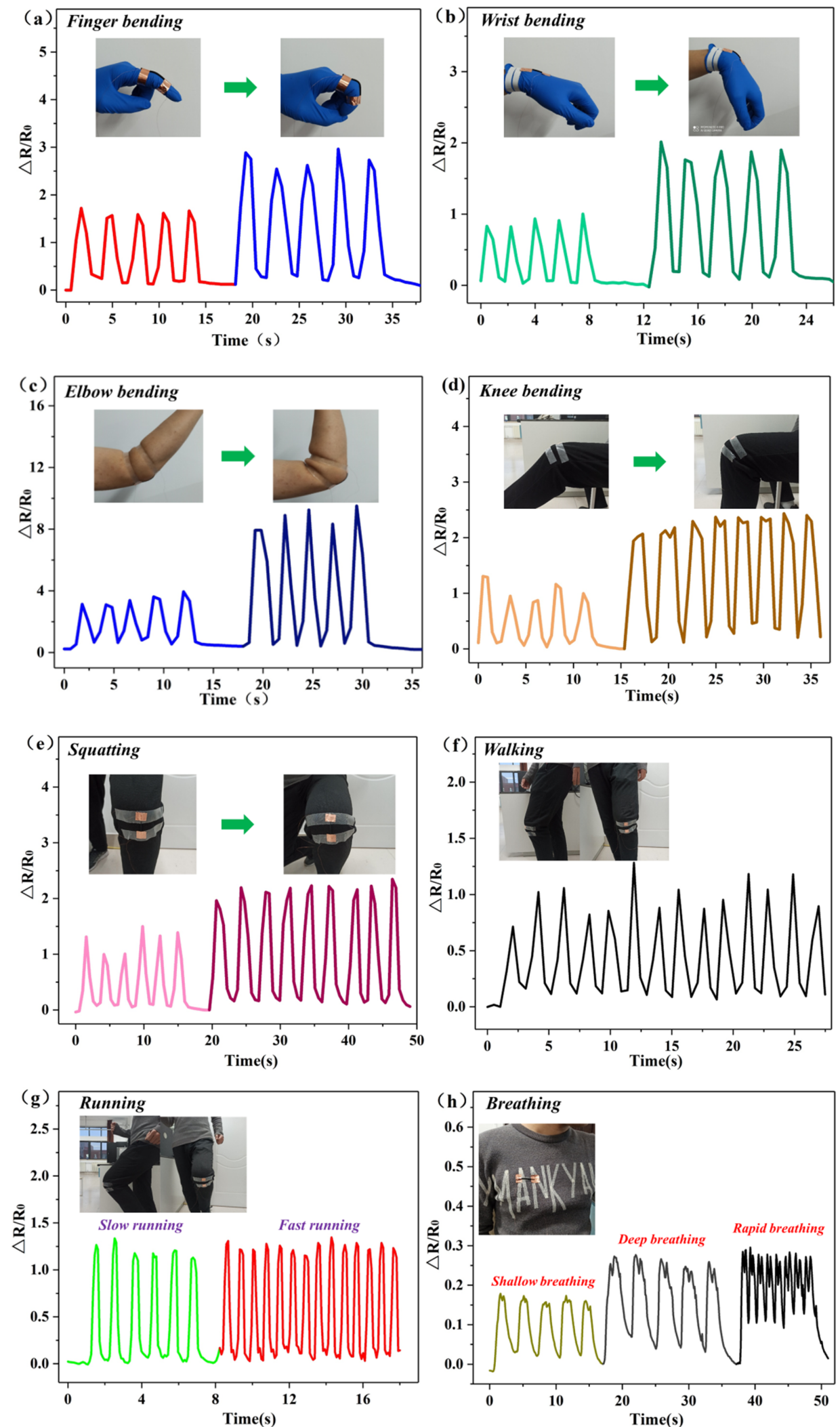

Figure 8. Monitoring of human motions using flexible strain sensors: the responses to human motions of (a) finger bending, (b) wrist bending, (c) elbow bending, (d) knee bending, (e) squatting, (f) walking, and $(\mathrm{g})$ running, and $(\mathbf{h})$ the responses to breathing. 
In addition, sensors were attached to the volunteers' chests to monitor the $\Delta R / R_{0}$ changes corresponding to shallow, deep, and rapid breathing. As shown in Figure $8 \mathrm{~h}$, the $\Delta R / R_{0}$ varies with the respiratory rate as the volunteer breathes. The graph indicates that the $\Delta R / R_{0}$ can change with the rate of respiration when the volunteer is breathing. Compared to shallow breathing, when the human body exhibits deep breathing, the $\Delta R / R_{0}$ displays a greater change. This is because deep breathing increases the strength of the body's breathing and the expansion of the chest, leading to an increase in strain sensor responses. The $\Delta R / R_{0}$ changes more frequently when the body breathes rapidly compared to normal breathing. At this time, the respiratory rate becomes stronger, causing the strain frequency of the strain sensor to increase. The test result is consistent with the actual situation, which also provides evidence for the abilities of the sensor to detect the physiological activities of the human body in the future.

\section{Conclusions}

In summary, by combining the conductive properties of CB and MWNTS with the high tensile properties of the porous structure of the PDMS substrate, a strain sensor with a unique structure was designed and developed in this study. The experimental results provide evidence which suggests that the porous structure can improve the tensile properties of the sensor, allowing the substrate to be stretched by up to $130 \%$. As CB and MWNTS combine to form a unique nano-composite conductive network structure, the sensor exhibits excellent characteristics, such as a high sensitivity, good linear response, and wide tensile range, as well as good reliability and repeatability. We successfully verified the feasibility of the sensor in terms of motion detection through various experiments. The experimental results show that the stress sensor designed using this kind of method not only performs desirably in terms of movement detection by deformation (such as the movement of fingers, wrists, elbows, knee joints, etc.), but is also capable of detecting the physiological activities of the body (such as the respiration intensity and frequency). Additionally, the simplicity, low cost, manufacturability, and easy integration of the sensor, prove that this design can provide a new feasible approach for motion detection in the field of wearable devices.

Author Contributions: Conceptualization, L.H., P.Z. and Y.L.; methodology, P.Z.; software, J.Z.; validation, P.Z., Y.C. and Y.Z.; formal analysis, P.Z.; investigation, Y.Z.; resources, L.H.; data curation, P.Z.; writing-original draft preparation, P.Z.; writing-review and editing, P.Z.; visualization, J.Z.; supervision, L.H.; project administration, L.H. All authors have read and agreed to the published version of the manuscript.

Funding: The study was supported by the "Major research program of national natural science foundation of China (Grant No. 91848206)"; the "Major scientific and technological innovation project of Shandong Province, China (Grant No. 2017CXGC0901)"; and the "Major basic research project of Shandong natural science foundation, China (Grant No. ZR2018ZC0436)".

Conflicts of Interest: The authors declare no conflicts of interest.

\section{References}

1. Gilshteyn, E.P.; Romanov, S.A.; Kopylova, D.S.; Savostyanov, G.V.; Anisimov, A.S.; Glukhova, O.E.; Nasibulin, A.G. Mechanically Tunable Single-Walled Carbon Nanotube Films as a Universal Material for Transparent and Stretchable Electronics. ACS Appl. Mater. Interfaces 2019, 11, 27327-27334. [CrossRef]

2. Zheng, X.; Wang, Q.; Luan, J.J.; Li, Y.; Wang, N. Patterned Metal/Polymer Strain Sensor with Good Flexibility, Mechanical Stability and Repeatability for Human Motion Detection. Micromachines 2019, 10, 472. [CrossRef]

3. Yu, H.; Lian, Y.L.; Sun, T.; Yang, X.N.; Wang, Y.; Xie, G.Z.; Du, X.S.; Gou, J.; Li, W.Z.; Tai, H.L. Two-Sided Topological Architecture on a Monolithic Flexible Substrate for Ultrasensitive Strain Sensors. ACS Appl. Mater. Interfaces 2019, 11, 43543-43552. [CrossRef]

4. Yan, C.Y.; Wang, J.X.; Kang, W.B.; Cui, M.Q.; Wang, X.; Foo, C.Y.; Chee, K.J.; Lee, P.S. Highly Stretchable Piezoresistive Graphene-Nanocellulose Nanopaper for Strain Sensors. Adv. Mater. 2014, 26, $2022-2027$. [CrossRef] 
5. Son, W.; Kim, K.B.; Lee, S.; Hyeon, G.; Hwang, K.G.; Park, W.J. Ecoflex-Passivated Graphene-Yarn Composite for a Highly Conductive and Stretchable Strain Sensor. J. Nanosci. Nanotechnol. 2019, 16, 6690-6695. [CrossRef] [PubMed]

6. Du, D.H.; Li, P.C.; Ouyang, J.Y. Graphene Coated Nonwoven Fabrics as Wearable Sensors. J. Mater. Chem. C 2016, 4, 3224-3230. [CrossRef]

7. Ma, Z.H.; Xu, R.; Wang, W.; Yu, D. A wearable, anti-bacterial strain sensor prepared by silver plated cotton/spandex blended fabric for human motion monitoring. Colloids Surf. A 2019, 582, 123918. [CrossRef]

8. Li, Q.; Li, J.; Tran, D.Q.; Luo, C.Q.; Gao, Y.; Yu, C.J.; Xuan, F.Z. Engineering of carbon nanotube/polydimethylsiloxane nanocomposites with enhanced sensitivity for wearable motion sensors. J. Mater. Chem. C 2017, 5, 11092-11099. [CrossRef]

9. Roh, E.; Hwang, B.-U.; Kim, D.; Kim, B.-Y.; Lee, N.-E. Stretchable, Transparent, Ultrasensitive, and Patchable Strain Sensor for Human-Machine Interfaces Comprising a Nanohybrid of CarbonNanotubes and Conductive Elastomers. ACS Nano 2015, 9, 6252-6261. [CrossRef] [PubMed]

10. Lee, C.J.; Park, K.H.; Han, C.J.; Oh, M.S.; You, B.; Kim, Y.-S.; Kim, J.-W. Crack-induced Ag nanowire networks for transparent, stretchable, and highly sensitive strain sensors. Sci. Rep. 2017, 7, 7959. [CrossRef] [PubMed]

11. Bauer, S.; Bauer-Gogonea, S.; Graz, I.; Kaltenbrunner, M.; Keplinger, C.; Schwödiauer, R. 25th Anniversary Article: A Soft Future: From Robots and Sensor Skin to Energy Harvesters. Adv. Mater. 2014, 26, 149-162. [CrossRef] [PubMed]

12. Chong, Y.S.; Yeoh, K.H.; Leow, P.L.; Chee, P.S. Piezoresistive strain sensor array using polydimethylsiloxane-based conducting nanocomposites for electronic skin application. SENSOR Rev. 2018, 38, 494-500. [CrossRef]

13. Trung, T.Q.; Lee, N.E. Flexible and Stretchable Physical Sensor Integrated Platforms for Wearable Human-Activity Monitoring and Personal Healthcare. Adv. Mater. 2016, 28, 4338-4372. [CrossRef]

14. Yin, F.G.; Lu, H.J.; Pan, H.; Ji, H.J.; Pei, S.; Liu, H.; Huang, J.Y.; Gu, J.H.; Li, M.Y.; Wei, J. Highly Sensitive and Transparent Strain Sensors with an Ordered Array Structure of AgNWs for Wearable Motion and Health Monitoring. Sci. Rep. 2019, 9, 2403. [CrossRef] [PubMed]

15. Yin, F.; Ye, D.; Zhu, C.; Qiu, L.; Huang, Y.A. Stretchable, Highly Durable Ternary Nanocomposite Strain Sensor for Structural Health Monitoring of Flexible Aircraft. Sensors 2017, 17, 2677. [CrossRef] [PubMed]

16. Chen, Y.; Wang, L.; Wu, Z.F.; Luo, J.C.; Li, B.; Huang, X.W.; Xue, H.G.; Gao, J.F. Super-hydrophobic, durable and cost-effective carbon black/rubber composites for high performance strain sensors. Compos. Part B Eng. 2019, 17, 107358. [CrossRef]

17. Liu, Y.W.; Zheng, H.J.; Liu, M.X. High performance strain sensors based on chitosan/carbon black composite sponges. Mater. Des. 2018, 141, 276-285. [CrossRef]

18. Yamada, T.; Hayamizu, Y.; Yamamoto, Y.; Yoshiki Yomogida, Y.; Ali Izadi-Najafabadi, A.; Futaba, D.N.; Hata, K. A stretchable carbon nanotube strain sensor for human-motion detection. Nat. Nanotechnol. 2011, 6, 296-301. [CrossRef]

19. Gao, Y.; Fang, X.L.; Tan, J.P.; Lu, T.; Pan, L.K.; Xuan, F.Z. Highly sensitive strain sensors based on fragmentized carbonnanotube/polydimethylsiloxane composites. Nanotechnology 2018, 29, 235501. [CrossRef]

20. Tian, H.; Shu, Y.; Cui, Y.L.; Mi, W.T.; Yang, Y.; Xie, D.; Ren, T.L. Scalable fabrication of high-performance and flexible graphene strain sensors. Nanoscale. 2014, 6, 699. [CrossRef]

21. Liu, H.B.; Xiang, H.C.; Wang, Y.; Li, Z.J.; Qian, L.W.; Li, P.; Ma, Y.C.; Zhou, H.W.; Huang, W. A Flexible Multimodal Sensor That Detects Strain, Humidity, Temperature, and Pressure with Carbon Black and Reduced Graphene Oxide Hierarchical Composite on Paper. ACS Appl. Mater. Interfaces 2019, 11, 40613-40619. [CrossRef] [PubMed]

22. Amjadi, M.; Pichitpajongkit, A.; Lee, S.; Ryu, S.; Park, I. Highly Stretchable and Sensitive Strain Sensor Based on Silver Nanowire-Elastomer Nanocomposite. ACS Nano. 2014, 8, 5154-5163. [CrossRef] [PubMed]

23. Li, W.; He, Y.; Xu, J.; Wang, W.Y.; Zhu, Z.T.; Liu, H. Preparation of Ag NWs and Ag NWs@PDMS stretchable sensors based on rapid polyol method and semi-dry process. J. Alloys Compd. 2019, 803, 332-340. [CrossRef]

24. Zheng, Y.J.; Li, Y.L.; Li, Z.Y.; Wang, Y.L.; Dai, K.; Zheng, G.Q.; Liu, C.T.; Shen, C.Y. The effect of filler dimensionality on the electromechanical performance of polydimethylsiloxane based conductive nanocomposites for flexible strain sensors. Compos. Sci. Technol. 2017, 139, 64-73. [CrossRef] 
25. Pei, Z.; Liu, Y.; Zhang, Q.; Zhao, D.; Wang, J.; Yuan, Z.Y.; Zhang, W.D.; Sang, S.B. Highly Sensitive, Stretchable Strain Sensor Based on Ag@COOH-Functionalized CNTs for Stroke and Pronunciation Recognition. Adv. Electron. Mater. 2019, 5, 1900227. [CrossRef]

26. Sun, H.L.; Dai, K.; Zhai, W.; Zhou, Y.J.; Li, J.W.; Zheng, G.Q.; Li, B.; Liu, C.T.; Shen, C.Y. A Highly Sensitive and Stretchable Yarn Strain Sensor for Human Motion Tracking Utilizing a Wrinkle-Assisted Crack Structure. ACS Appl. Mater. Interfaces 2019, 11, 36052-36062. [CrossRef]

27. Gao, J.F.; Li, B.; Huang, X.W.; Wang, L.; Lin, L.W.; Wang, H.; Xue, H.G. Electrically conductive and fluorine free superhydrophobic strain sensors based on $\mathrm{SiO}$ /graphene-decorated electrospun nanofibers for human motion monitoring. Chem. Eng. J. 2019, 373, 298-306. [CrossRef]

28. Guo, X.H.; Huang, Y.; Zhao, Y.N.; Mao, L.D.; Gao, L.; Pan, W.D.; Zhang, Y.G.; Liu, P. Highly stretchable strain sensor based on SWCNTs/CB synergistic conductive network for wearable human-activity monitoring and recognition. Smart Mater. Struct. 2017, 26, 095017. [CrossRef]

29. Qiu, J.; Guo, X.H.; Chu, R.; Wang, S.L.; Zeng, W.; Qu, L.; Zhao, Y.N.; Yan, F.; Xing, G.Z. Rapid-Response, Low Detection Limit, and High-Sensitivity Capacitive Flexible Tactile Sensor Based on Three-Dimensional Porous Dielectric Layer for Wearable Electronic Skin. ACS Appl. Mater. Interfaces 2019, 11, 40716-40725. [CrossRef]

30. Yan, T.; Zhou, H.; Niu, H.T.; Shao, H.; Wang, H.X.; Pan, Z.J.; Lin, T. Highly sensitive detection of subtle movement using a flexible strain sensor from helically wrapped carbon yarns. J. Mater. Chem., C 2019, 7, 10049-10058. [CrossRef]

31. Liu, P.; Pan, W.D.; Liu, Y.; Liu, J.; Xu, W.R.; Guo, X.H.; Liu, C.X.; Zhang, Y.G.; Ge, Y.J.; Huang, Y. Fully flexible strain sensor from core-spun elastic threads with integrated electrode and sensing cell based on conductive nanocomposite. Compos. Sci. Technol. 2018, 159, 42-49. [CrossRef]

32. Wang, Y.L.; Jia, Y.Y.; Zhou, Y.J.; Wang, Y.; Zheng, G.Q.; Dai, K.; Liu, C.T.; Shen, C.Y. Ultra-stretchable, sensitive and durable strain sensors based on polydopamine encapsulated carbon nanotubes/elastic bands. J. Mater. Chem., C 2018, 6, 8160-8170. [CrossRef]

33. Zheng, Y.J.; Li, Y.L.; Dai, K.; Wang, Y.; Zheng, G.Q.; Liu, C.T.; Shen, C.Y. A highly stretchable and stable strain sensor based on hybrid carbon nanofillers/polydimethylsiloxane conductive composites for large human motions monitoring. Compos. Sci. Technol. 2018, 156, 276-286. [CrossRef]

34. Huang, Y.; Hao, C.; Liu, J.; Guo, X.H.; Zhang, Y.Y.; Liu, P.; Liu, C.X.; Zhang, Y.G. Highly stretchable, rapid-response strain sensor based on SWCNTs/CB nanocomposites coated on rubber/latex polymer for human motion tracking. Sensor Rev. 2019, 39, 233-245. [CrossRef]

35. Wang, L.H.; Ding, T.H.; Wang, P. Influence of carbon black concentration on piezoresistivity for carbon-black-filled silicone rubber composite. Carbon 2009, 47, 3151-3157.

36. Li, Y.B.; Shang, Y.Y.; He, X.D.; Peng, Q.Y.; Du, S.Y.; Shi, E.Z.; Wu, S.T.; Li, Z.; Li, P.X.; Cao, A.Y. Overtwisted, Resolvable Carbon Nanotube Yarn Entanglement as Strain Sensors and Rotational Actuators. ACS Nano. 2013, 7, 8128-8135. [CrossRef]

37. Zhang, S.J.; Zhang, H.L.; Yao, G.; Liao, F.Y.; Gao, M.; Huang, Z.L.; Li, K.Y.; Lin, Y. Highly stretchable, sensitive, and flexible strain sensors based on silver nanoparticles/carbon nanotubes composites. J. Alloys Compd. 2015, 652, 48-54. [CrossRef]

38. Zhang, R.F.; Peng Pan, P.; Dai, Q.L.; Yang, X.P.; Yang, Z.C.; Wei, J.; Liu, J.; Yuan, Q.P. Sensitive and wearable carbon nanotubes/carbon black strain sensors with wide linear ranges for human motion monitoring. J. Mater. Sci. - Mater. Electron. 2018, 29, 5589-5596. [CrossRef]

(C) 2020 by the authors. Licensee MDPI, Basel, Switzerland. This article is an open access article distributed under the terms and conditions of the Creative Commons Attribution (CC BY) license (http://creativecommons.org/licenses/by/4.0/). 